\title{
Surface Energetics of Mycobacterium Tuberculosis - Macrophage Interactions
}

\author{
${ }^{1}$ Chukwuneke J. L., ${ }^{1}$ Achebe C. H., ${ }^{2}$ Ejiofor O. S. and ${ }^{3}$ Sinebe J. E. \\ ${ }^{1}$ Department of Mechanical Engineering, Nnamdi Azikiwe University, Awka, Nigeria \\ ${ }^{2}$ Department of Paediatrics, Anambra State University Teaching Hospital, Awka, Nigeria \\ ${ }^{3}$ Department of Mechanical Engineering, Delta State University, Oleh, Nigeria \\ jl.chukwuneke@unizik.edu.ng
}

\begin{abstract}
The surface thermodynamics of M-Tb/HIV-macrophage interactions were studied using the Hamaker coefficient concept as a surface energetics tool in determining the interaction processes. The surface interfacial energies were explained using van der Waals concept of particle - particle interactions. The method involved sputum sample collection, mycobacterium and macrophages structural studies, and the study of the mechanism of interaction of the bacterium and the macrophage. Twenty samples each of infected, uninfected and M-Tb/HIV co-infected sputum were collected. Each specimen was screened to determine the infection status using GeneXpert and Ziehl-Neelsen staining methods. The absorbance, $\bar{a}$, values of each specimen, for wavelength range of 230 - 950nm were measured using digital Ultraviolet Visible Spectrophotometer. From the absorbance data the variables (e.g. dielectric constant, etc.) required for computations were derived. MatLab software tools were employed in the mathematical analysis. The Hamaker constants, combined Hamaker coefficients and absolute combined Hamaker coefficients were obtained. The values of $A_{132 a b s}=0.21631 \times 10^{-21}$ Joule and $\tilde{A}_{132 a b s}=0.18825 \times 10^{-}$ ${ }^{21}$ Joule were obtained for $\mathrm{M}-\mathrm{Tb}$ and $\mathrm{M}$-Tb/HIV infected sputum respectively. The implication of this result is the positive value of the absolute combined Hamaker coefficient which entails net positive van der waals forces demonstrating an attraction between $\mathrm{M}-\mathrm{Tb}$ and the macrophage. This however, implies that infection is very likely to occur. It was also shown that in the presence of HIV, the interaction energy is reduced by $13 \%$ confirming adverse effects observed in HIV patients suffering from tuberculosis. The lower value for the combined Hamaker coefficient $A_{131 a b s}=10165 \times 10^{-21}$ Joule for the uninfected sputum samples is an indicator that a negative Hamaker coefficient is realistic. The desired outcome is that the bacteria do not adhere to the macrophage to avoid penetration, in which case a condition for rendering combined Hamaker coefficient negative is required. Thus, condition was sought for repulsion to occur and that condition was based on the value of $A_{33}$ that would render the absolute combined Hamaker coefficient negative. To achieve the condition of $A_{33}$ above, possible additive(s) in form of drugs to the sputum should be required.
\end{abstract}

Keywords: Absorbance, Dielectric Constant, Energetics, Hamaker Coefficient, Lifshitz Formula, Macrophage, Mycobacterium Tuberculosis, Surface Interfacial, van der Waals Forces, Wavelength 


\section{Introduction}

Tuberculosis (Tb) is an airborne disease caused by the bacterium Mycobacterium $\mathrm{Tb}(\mathrm{M}-\mathrm{Tb})$. This causative agent of $\mathrm{Tb}$, is one of the world's most devastating human pathogens. The contagious disease is transmitted through air and most commonly affects the lungs, which is responsible for more than $75 \%$ of cases (De Souza, 2006). The initial infection usually occurs in the lungs and in most cases is controlled by the immune system. Even after successful control of primary $\mathrm{Tb}$ infection, some bacilli remain in a non- or slowing replicating state, termed latent Tb infection (LTbI). Latently infected individuals have a $10 \%$ risk of developing the disease in their lifetime (WHO, 2012), which constitutes a huge global reservoir of infection and a continuous threat of disease transmission.

Tuberculosis (Tb) is the leading infectious killer worldwide; it is estimated that one-third of the world's population is currently infected with Tb, and it results in 3 million deaths every year (WHO, 2005). Human Immunodeficiency Virus (HIV) is a strong risk factor for developing active $\mathrm{Tb}$, and $\mathrm{Tb}$ is the leading cause of death among HIV-positive individuals. Out of 4.1 million AIDS deaths in 2004, 13\% can be attributed to Tb (WHO, 2005).

The highest incidence of disease is in Sub-Saharan Africa, in part due to association with Human Immunodeficiency Virus (HIV) (Nunn et al, 2005; Maartens and Wilkinson, 2007), which has fuelled dramatic rises in incidence of the disease in many countries. [Globally, Tb is the proximate cause of many HIV-related deaths, particularly in Africa (Corbett et al, 2006)]. Even in many countries where its overall incidence is low, $\mathrm{Tb}$ remains a problem.

A review of the Tb cases reported by 134 countries between 1998 and 2007 found that only 35 had per capita rates of decline exceeding $5 \%$ per year (Adeeb et al, 2013). Surveillance and mathematical modeling suggests that the total Tb incidence per capita is falling at an estimated $1 \%$ per year, a finding that indicates that the global incidence rate will decrease by 2015. The world's population is growing at about $2 \%$ per year, and thus the total number of new TB cases remains on the rise (WHO, 2009). This finding reveals the relative failure of the existing management strategies for $\mathrm{Tb}$ and the inadequate effectiveness of public health systems, mainly in underdeveloped countries.

In spite of the availability of some effective treatment, the steady emergence of multi-drug resistant (MDR), extremely drug-resistant (XDR) and totally drug-resistant strains (TDR) forms of TB is a cause of concern. Globally MDR-Tb accounts for roughly $3.6 \%$ of all Tb cases, but accounts for up to $28 \%$ of Tb cases in some regions (De Souza, 2006; Gonzalez-Juarrero et al, 2001). The emergence of MDR, XDR and $T D R T b$ is very worrying due to the increasing difficulty of treating these forms of $T b$ and rendering even the frontline drugs inactive.

In addition, drugs such as Rifampicin have high levels of adverse effects making them prone to patient incompliance. Another important problem with most of the anti-mycobacterials is their inability to act upon latent forms of the bacillus. To compound the problems further, the deadly associations between the HIV and TB makes the treatment of co-infected patients even more challenging (Nunes et al, 2011; Gonzalez-Juarrero et al, 2001). Much research has been and is still on, on the subject with a cure not yet in view. The choice to approach this issue via the vehicle of surface thermodynamics energetics against the conventional clinical methods is a novel one. The role of surface properties in this biological process will be established. 
Chukwuneke J. L., Achebe C. H., Ejiofor O. S. and Sinebe J. E.; Surface Energetics of Mycobacterium Tuberculosis-Macrophage Interactions. Journal of Biomedical Engineering and Medical Imaging, Volume 2, No 6, December (2015), pp 49-61

In this study, it is of interest to understand how the mycobacterium tuberculosis (M-TB) interacts with the macrophage. The process involves the bacterium (modeled as a particle) in a liquid medium (sputum) attaching itself on the surface of the macrophage (another particle), penetrating and probably destroying it. The condition under which bacterium attachment on the surface of the macrophage does not take place will be sought, even in the presence of HIV with its destructive effect.

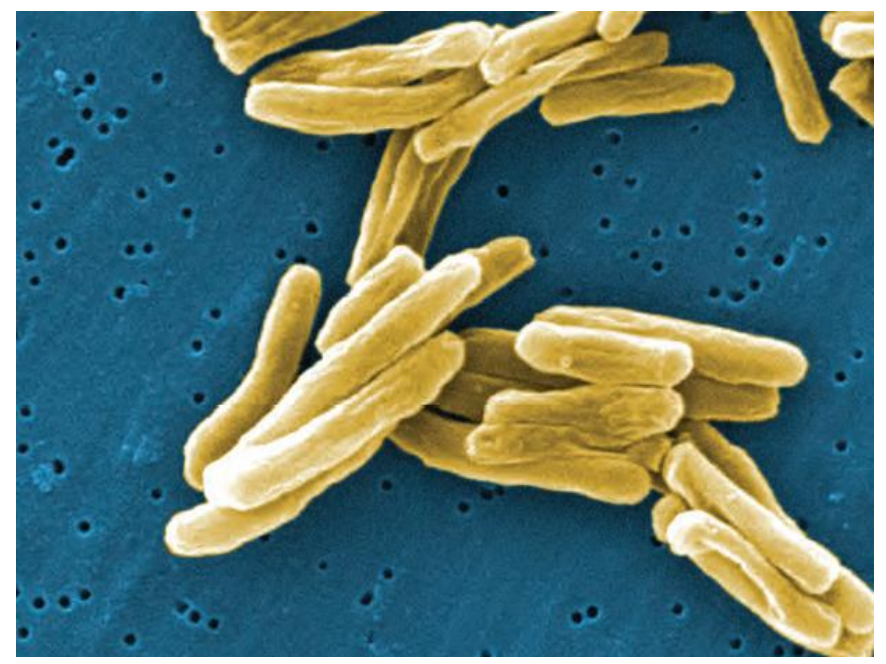

Figure.1: Mycobacterium Tuberculosis

The Lifshiftz derivation for van der Waals forces is used to model the interaction to predict the nature of interaction between two solid particles suspended in a liquid medium (Sputum). Hamaker Coefficient is a significant thermodynamic energetics tool used in determining the interaction processes and will be used in these M-Tb - Macrophage interactions. It is a well-known fact that surface property determination of interacting particles leads to the further understanding of the mechanism of interactions. A common area of contact is established once two solid particles meet each other. In such process, a certain portion of each particle gets displaced through work. Work responsible for the displacement of a unit area is known as surface free energy. The consecutive impact on the surface is known as surface thermodynamic energetics effects. In this particular study similar concepts have been implemented to characterize the $\mathrm{M}-\mathrm{Tb}$ - macrophage interactions with the sputum as the intervening medium.

\section{Methodology}

\subsection{Sample Collection, Preparation and Measurement}

The methodology involved sputum sample collection, mycobacterium and macrophages structural studies, mycobacterium tuberculosis screening, and the study of the mechanism of interactions of the bacterium and the macrophage. Twenty samples each of infected, uninfected and M-Tb/HIV co-infected sputum were collected from Anambra State University Teaching Hospital (ANSUTH) Awka. Each specimen was screened to determine the infection status using GeneXpert and Ziehl-Neelsen staining method thus giving a total of sixty sputum samples from different individuals. 


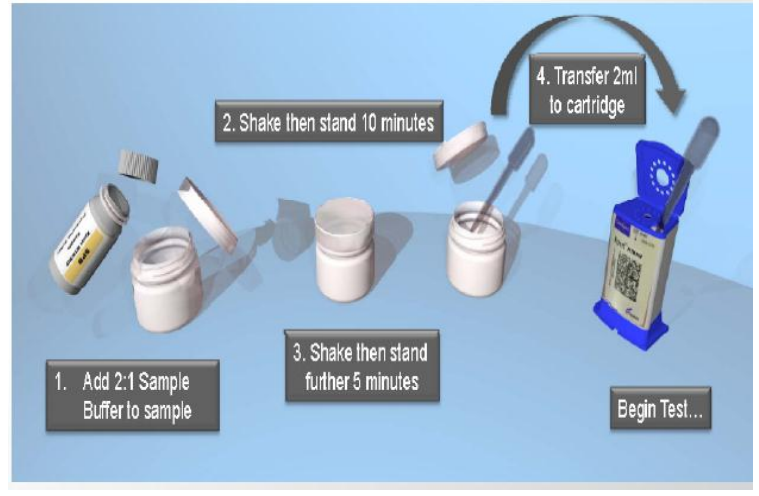

Figure. 2: Sample Preparation: direct sputum

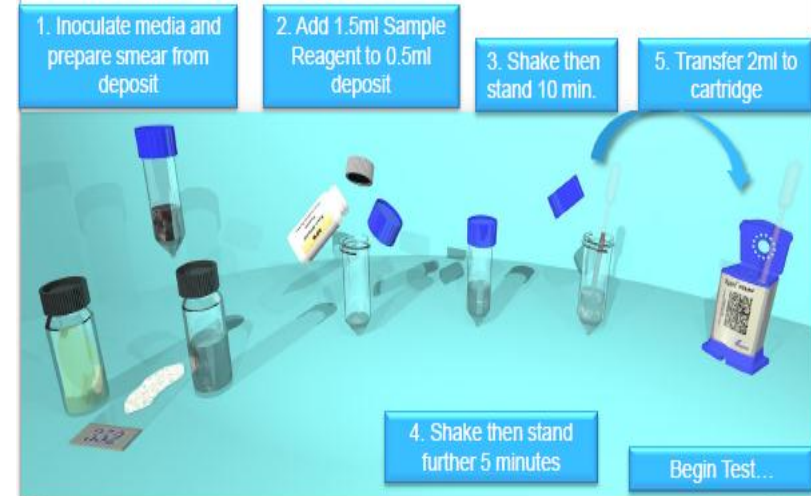

Figure. 3: Sample Processed Sputum Sediment

The glass slides of $25.4 \mathrm{~mm} \times 76.2 \mathrm{~mm} \times 1.2 \mathrm{~mm}$ were prepared for test surfaces. A dropper was used to draw each of the Sputum samples from the container and smeared carefully on a slide to ensure even distribution of the sputum samples on the slides. The slide preparations and sample smearing were done at the same laboratory (Chest Clinic/Laboratory, Anambra State University Teaching Hospital, Awka). The samples were allowed to dry naturally at room temperature because exposing the prepared slides to the sun is likely to cause oxidation and the surface energy might be increased unconditionally. All the well prepared and dried surfaces were covered with microscopic cover slip and the absorbance, $\bar{a}$, values of each specimen, for wavelength range of 230 - 950nm were measured using digital Ultraviolet Visible Spectrophotometer (UV/VIS MetaSpecAE1405031Pro). The data generated and the various equations governing the relationship among the variables were used in calculating values for the reflectance, $R$, transmittance, $T$, refractive index, $n$, and the dielectric constant, $\varepsilon$. MatLab software tools were employed in the mathematical analysis of the data generated from the absorbance values.

\subsection{Mathematical Applications to the System}

To be able to use the absorbance data to calculate the Hamaker coefficients using the Lifshitz theorem, there is a need to evaluate the dielectric constant $\varepsilon$ of the equation. Some relevant equations are required. From the information of light absorbance, reflection and transmittance, it could be seen that;

$$
\bar{a}+T+R=1
$$

Where; $\bar{a}$ is absorbance, $T$ is transmittance, and $R$ is reflectance. Also, from the information of light absorbance and transmittance;

$$
T=\exp ^{-\bar{a}}
$$

With the values of à determined from absorbance experimental results, and substituting the values of $\bar{a}$ into Eq. (2) to obtain T; R could easily be derived by substituting the values of à and T into Eq.(1).

The value for the refractive index, $n$ employing the mathematical relation (Robinson, 1952);

$$
n=\left[\frac{1-R^{1 / 2}}{1+R^{1 / 2}}\right]
$$

Value for the extinction coefficient, $k$ is obtained from the equation; 


$$
k=\left[\frac{\alpha \lambda \times 10^{-9}}{4 \pi}\right]
$$

Where; $\alpha$ is the absorption coefficient defined as follows;

$$
\alpha=\left[\frac{\bar{a}}{\lambda \times 10^{-9}}\right]
$$

Substituting the value, $\alpha$ of Eq. (5) into Eq. (4);

$$
k=\left[\frac{\bar{a}}{4 \pi}\right]
$$

The dielectric constant, $\varepsilon$ could thus be given by the formula (Charles, 1996);

For the real part;

$$
\varepsilon_{1}=n_{1}^{2}-k^{2}
$$

For the imaginary part;

$$
\varepsilon_{2}=2 n_{2} k
$$

With these values, it is possible to determine $A_{i j}$ using the relevant equations to determine $A_{11}$.

$$
A_{11}=2.5\left[\frac{\varepsilon_{10}-1}{\varepsilon_{10}+1}\right]^{2}=2.5\left[\frac{n_{1}^{2}-1}{n_{1}^{2}+1}\right]^{2}
$$

This gives a value to the Hamaker constant $A_{11}$, and by extension to other Hamaker constants $A_{22}$ and $A_{33}$.

For a combination of two disimilar materials (i.e. macrophage, 1 and the bacteria, 2) with the gap between 1 and 2 filled with sputum as the medium 3 (see Fig. 4).

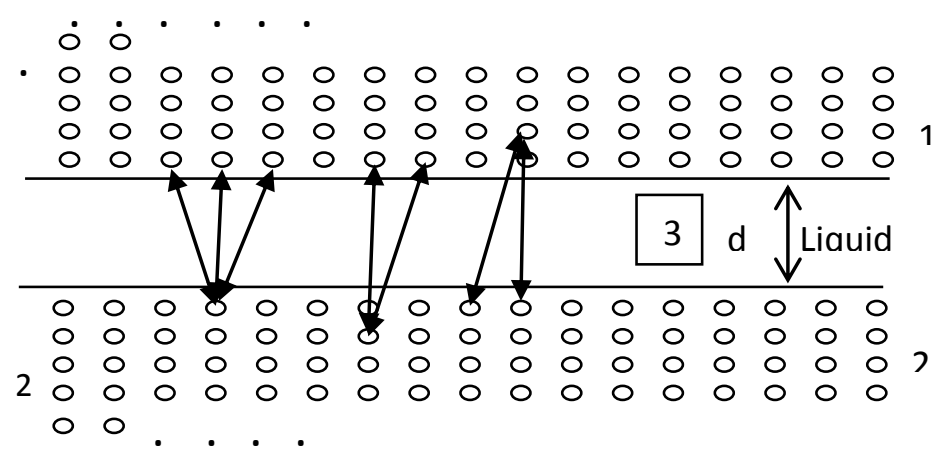

Figure. 4: Schematic representation of interaction of two solid bodies, depicted by 1 and 2 which are eventually isolated by $d$, in liquid 3 .

Thus, the combined Hamaker coefficient, $A_{131}, A_{232}$ and $A_{132}$ could be gotten from the relations [Hamaker, 1937; Visser, 1981; Chukwuneke et al, 2015]; 


$$
A_{131}=A_{11}+A_{33}-2 A_{13}
$$

$$
\text { Alternatively; } \quad \begin{aligned}
A_{131} & =\left(\sqrt{A_{11}}-\sqrt{A_{33}}\right)^{2} \\
A_{232} & =A_{22}+A_{33}-2 A_{23}
\end{aligned}
$$

$$
\text { Alternatively; } \quad \begin{aligned}
A_{232} & =\left(\sqrt{A_{22}}-\sqrt{A_{33}}\right)^{2} \\
A_{132} & =A_{12}+A_{33}-A_{13}-A_{23}
\end{aligned}
$$

$$
\text { Alternatively; } \quad A_{132}=\left(\sqrt{A_{11}}-\sqrt{A_{33}}\right)\left(\sqrt{A_{22}}-\sqrt{A_{33}}\right)
$$

Equation (12b) shows that, for a three-component system involving three different materials, 1, 2 and 3, $\mathrm{A}_{132}$ can become negative;

$$
A_{132} \prec 0
$$

When;

$$
\begin{gathered}
\sqrt{A_{11}} \succ \sqrt{A_{33}} \text { and } \sqrt{A_{22}} \prec \sqrt{A_{33}} \\
\sqrt{A_{11}} \prec \sqrt{A_{33}} \prec \sqrt{A_{22}}
\end{gathered}
$$

Hamaker's approach to the interaction between condensed bodies from molecular properties called microscopic approach has limitations. This is true against the backdrop of its neglect of the screening effect of the molecules which are on the surface of two interacting bodies as regards the underlying molecules. Therefore, Hamaker's approach is regarded as an over simplification.

A system containing two planes could be considered for computing the free energy of interaction. This can be done for semi-infinite, parallel bodies belonging to material 1 and 2 isolated by material 3, bearing thickness d (refer fig.4) provided in the following section (Hamaker, 1937; Lifshitz et al, 1961).This is calculated by the following equation:

$$
\Delta F_{132}(d)=\left[-\frac{A_{182}}{12 \pi d^{2}}\right]
$$

In this, $A_{132}$ refers to the Hamaker coefficient for a respective system.

Considering nominal isolation distance $d_{0}$, and $\mathrm{Eq}(16)$ as valid for such a small distance, the Hamaker coefficient should be expressed as (Chukwuneke et al, 2015; Hamaker, 1937; Lifshitz et al, 1961):

$$
A_{132}=-12 \pi d_{0}^{2} \Delta F^{a d h}\left(d_{0}\right)
$$

The Hamaker coefficient $A_{132}$ for the interactions between two different bodies in a liquid can be calculated from Eq.(17) once the free energy of adhesion between the two bodies is known or through the pair-wise additivity approach as originally proposed by (Hamaker, 1937) or by the macroscopic approach of (Lifshitz et al, 1961). Influence of neighbouring atoms remains major hurdle during the pairwise summation computing between various molecular interactions. In case of highly disperse media 
Chukwuneke J. L., Achebe C. H., Ejiofor O. S. and Sinebe J. E.; Surface Energetics of Mycobacterium Tuberculosis-Macrophage Interactions. Journal of Biomedical Engineering and Medical Imaging, Volume 2, No 6, December (2015), pp 49-61

such influence is insignificant, for instance, gases whereas for condensed media it is important (Visser, 1981).

As the actual material atomic structures are overlooked, the Lifshitz method is suitable in certain cases. In this method, bulk material properties are considered for calculation of interactions between the macroscopic bodies. Properties like refractive indices and dielectric permittivity $\varepsilon(i \zeta)$ are considered for such calculations.Dielectric permittivity represents the microscopic polarizability as a manifested macroscopic property for the constant atoms belonging to certain materials. The Hamaker coefficient represents the macroscopic resultant for the interactions happened due to the atom polarizations in a material (Hough and White, 1987).

Following Lifshitz theory, the Hamaker coefficient is represented as follows:

$$
A_{i k j}=\frac{3}{4} \pi \hbar \int_{0}^{\infty}\left[\frac{\varepsilon_{i}(i \zeta)-\varepsilon_{k}(i \zeta)}{\varepsilon_{i}(i \zeta)+\varepsilon_{k}(i \zeta)}\right]\left[\frac{\varepsilon_{j}(i \zeta)-\varepsilon_{k}(i \zeta)}{\varepsilon_{j}(i \zeta)+\varepsilon_{k}(i \zeta)}\right] d \zeta
$$

Where, $\varepsilon_{\mathrm{j}}(\mathrm{i} \zeta)$ refers to the dielectric constant of a specific material $\mathrm{j}$, this is considered through the

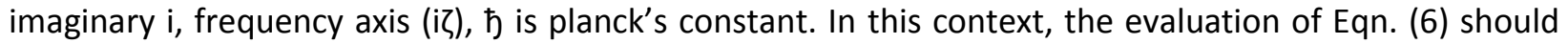
result in equivalent value with the thermodynamic free energy of adhesion, provided in Eqn. (19):

$$
\Delta \mathrm{F}_{132}{ }^{\text {adh }}\left(\mathrm{d}_{\mathrm{o}}\right)=\gamma_{\mathrm{ps}}-\gamma_{\mathrm{pl}}-\gamma_{\mathrm{sl}}
$$

The molecular contact was maintained at $(d=0)$. Interestingly, constituent molecule numbers are of finite size and for that it is not possible to attain $\mathrm{d}=0$ for two macroscopic surface. Therefore, whenever the surfaces attain a distance $d_{a}$, molecular contacts are considered. The divergences according to Lifshitz theory are eliminated by the parameter $\mathrm{d}_{\mathrm{o}}$.

Thus, the Hamaker coefficient or the Lifshitz-van der Waals constant $A_{132}$ may result in negative. In certain condition, the contact between the interacting particles will be hampered due to the repulsive (electrostatic) force originated. The resultant effect in certain cases remains as repulsion instead of attraction for the considered particles. The Hamaker coefficient and the interfacial free energies are connected through the following equation:

$$
A_{132}=-12 \pi d_{\circ}^{2}\left(\gamma_{12}-\gamma_{13}-\gamma_{23}\right)
$$

This equation has been derived through combining Eqn. (17) with Eqn. (19).

For the issue of self-interaction of a particle Eqn. (8) should be considered;

$$
A_{i j}=\frac{3}{4} \pi \hbar \int_{0}^{\infty}\left[\frac{\varepsilon_{i}(i \zeta)-\varepsilon_{j}(i \zeta)}{\varepsilon_{i}(i \zeta)+\varepsilon_{j}(i \zeta)}\right]^{2} d \zeta
$$

\section{Results and Discussion}

The raw data obtained for both $\mathrm{M}-\mathrm{Tb}$ and M-Tb/HIV positive and negative sputum samples were collated. This paved the way for the data analysis. However, since extinction coefficient " $k$ ", absorption coefficient " $\alpha$ " and dielectric constant " $\varepsilon$ " are obtained as functions of wavelength $\lambda$, an integration of 
Eq. (18) will give a more accurate value. The data on absorbance obtained as a function of wavelength are plotted on figs $5,6,7,8$, and 9 .

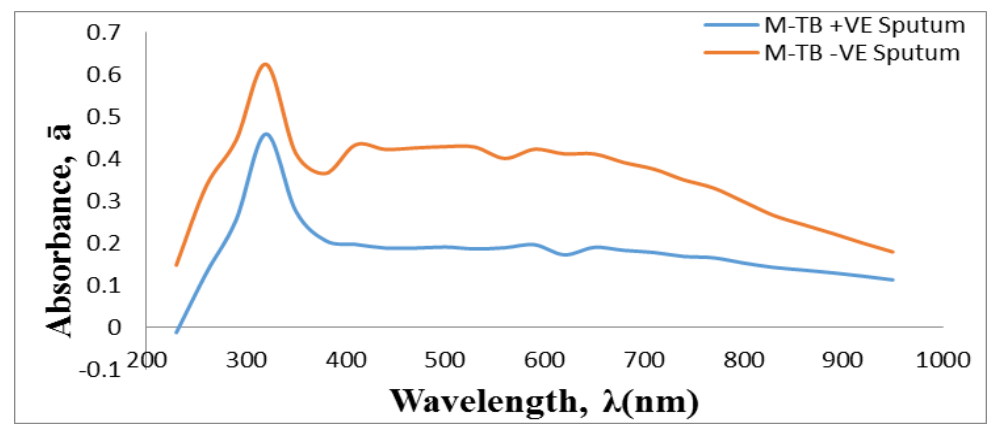

Figure.5: Variation of Average Absorbance, $\bar{a}$ with Wavelength, $\lambda$ for Twenty Samples of M-Tb Infected and Uninfected Sputum

Figure 5 shows a peak absorbance value of greater than 0.60 and 0.45 for M-Tb negative and positive sputum respectively were recorded at wavelength of $320 \mathrm{~nm}$. This peak value falls within the visible range of ultraviolet radiation which is between $300-600 \mathrm{~nm}$. This is important as a reference point in the study of the infection mechanism and may be of importance in determining the critical Hamaker coefficient that favours repulsion between the bacterium and the macrophage. It could be well-known that the infected $\mathrm{M}$-Tb sputum has lower absorbance values than the uninfected ones.

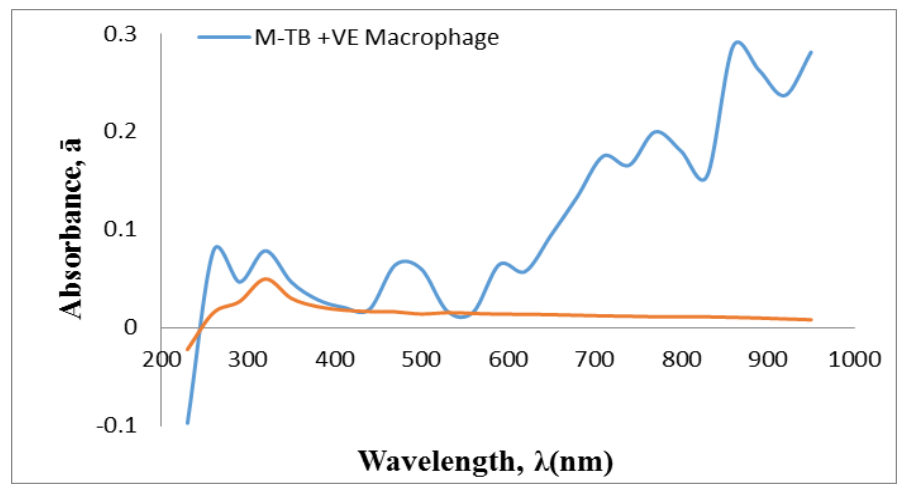

Figure.6: Variation of Average Absorbance, $\bar{a}$ with Wavelength, $\lambda$ for Twenty Samples of M-Tb Infected and Uninfected Macrophage

Figure 6 shows a peak absorbance value of greater than 0.07 and 0.04 for M-Tb positive and negative macrophages respectively were recorded at wavelength of $320 \mathrm{~nm}$ which corresponds exactly with that of the figure 5. It is interesting though, that at the lower wavelength of $230 \mathrm{~nm}$ some negative absorbance values were recorded. The absorbance values of both the M-Tb positive and negative Macrophages were increasing with increase in wavelength. This is the opposite of the result obtained with the M-Tb sputum samples. This may be explained away by the fact of a higher energy level of these cells. The M-Tb infected macrophages gave higher absorbance values than the M-Tb uninfected macrophages. This is a clear indication that infection had occurred and shows the alteration in absorbance values due to M-Tb infection. 
Chukwuneke J. L., Achebe C. H., Ejiofor O. S. and Sinebe J. E.; Surface Energetics of Mycobacterium Tuberculosis-Macrophage Interactions. Journal of Biomedical Engineering and Medical Imaging, Volume 2, No 6, December (2015), pp 49-61

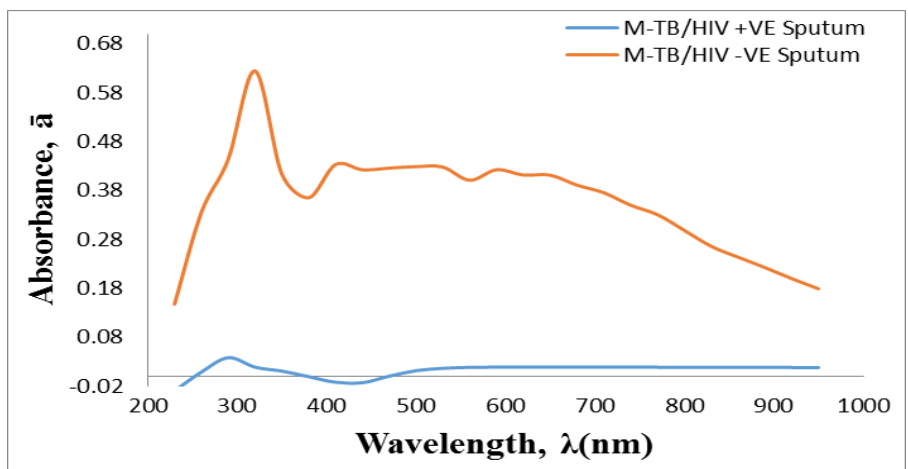

Figure.7: Variation of Average Absorbance, $\bar{a}$ with Wavelength, $\lambda$ for Twenty Samples of M-Tb/HIV Coinfected and M-Tb/HIV Uninfected Sputum

Figure 7 reveals an interesting pattern for both M-Tb/HIV positive and negative sputum. The absorbance of the M-Tb/HIV co-infected sputum samples systematically increased as the wavelength increased until a critical wavelength of $290 \mathrm{~nm}$, where peak absorbance values of greater than 0.60 and 0.06 for $M$ $\mathrm{Tb} / \mathrm{HIV}$ negative and positive sputum respectively were accomplished. The trend here shows that the uninfected sputum reveals higher absorbance values at all wavelengths. This indicates that a shift in the energy equation of the system is tenable by some alteration to the sputum as an intervening medium in the M-Tb/HIV - Macrophage interaction. It then suggests a possibility of attaining repulsion between the $\mathrm{M}-\mathrm{Tb} / \mathrm{HIV}$ and the Macrophage cells by some additives to the sputum.

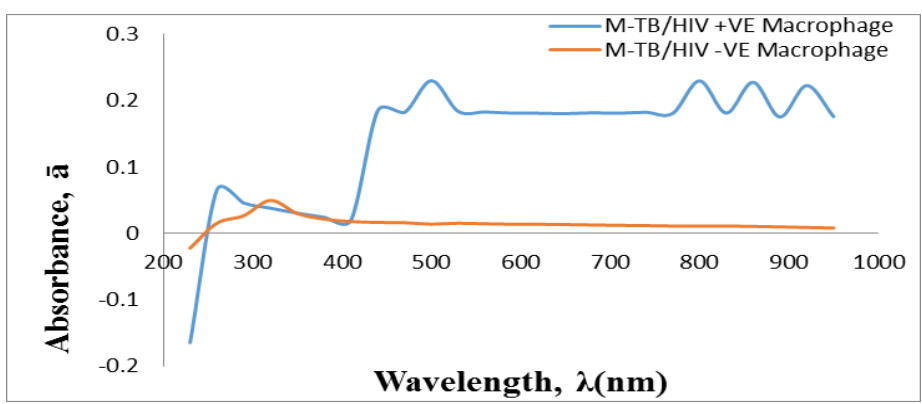

Figure.8: Variation of Average Absorbance, $\bar{a}$ with Wavelength, $\lambda$ for Twenty Samples of M-Tb/HIV Coinfected and Uninfected Macrophage

Figure 8 followed similar pattern as that of Fig. 6 for uninfected macrophage with the peak values occurring at the wavelength of 320nm. The absorbance values of the M-Tb/HIV positive Macrophages were increasing with increase in wavelength. This is the opposite of the result obtained with the sputum. The M-Tb/HIV coinfected macrophages also gave higher absorbance values than the uninfected ones at wavelengths greater than $400 \mathrm{~nm}$. This may be explained away by the fact of a higher energy level of these cells. This is a clear indicator that bacteria ingestion had occurred and shows the alteration in absorbance values due to M-Tb/HIV infection. 


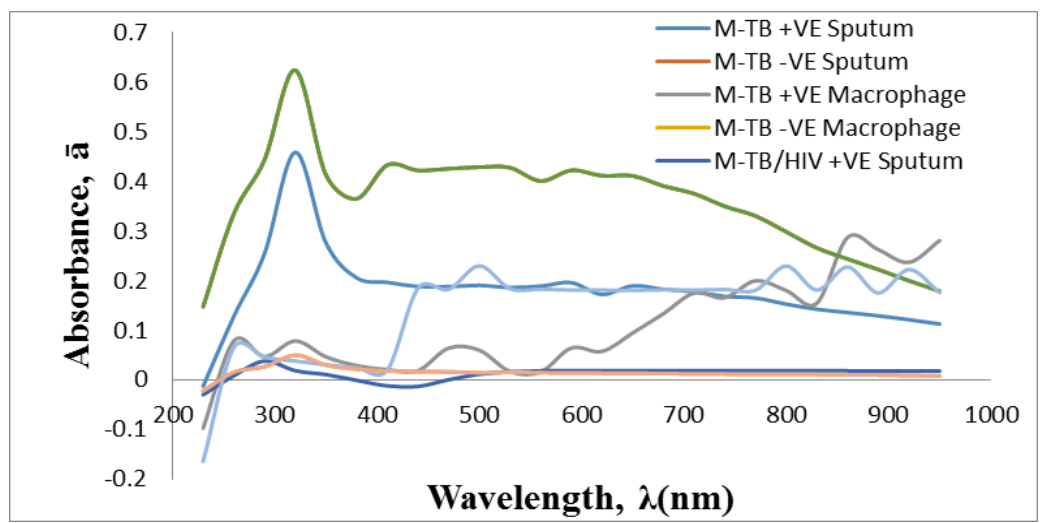

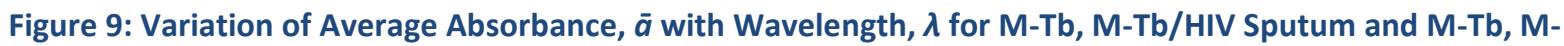
Tb/HIV Macrophages

Figure 9 reveals the disparity between the peak absorbance values of $\mathrm{M}$-Tb/HIV positive and negative sputum samples respectively, and this shows an indication of how the bacteria affect the properties of sputum.

Eq.(21) was used to obtain for each interacting system, $A_{i j}\left(A_{11}, A_{22}, A_{33}, A_{12}, A_{13}, A_{23}\right)$ by approximate change of variables. MatLab computation tools were used. This involved the numerical integration of Eq.(21) for each wavelength from 230 to 950 for all the twenty samples in each category. Applying Lifshitz derivation for van der Waals forces as in Eq.(18), The absolute value for the Hamaker coefficient could be derived by obtaining the mean of all the $A_{132}, A_{131}$, and $A_{232}$ values got from the Lifshitz relation.

Table 1: Values of the Hamaker Constants and Hamaker Coefficients for the Infected and Uninfected M-TB Sputum Samples

\begin{tabular}{|c|c|c|c|c|}
\hline \multirow{2}{*}{$\begin{array}{c}\text { Variable } \\
\left(\times 10^{-21} \text { Joule }\right)\end{array}$} & \multicolumn{2}{|c|}{ Infected Sputum } & \multicolumn{2}{c|}{ Uninfected Sputum } \\
\cline { 2 - 5 } & $\begin{array}{c}\text { Peak } \\
\text { Value }\end{array}$ & $\begin{array}{c}\text { Absolute } \\
\text { Value }\end{array}$ & $\begin{array}{l}\text { Peak } \\
\text { Value }\end{array}$ & Absolute Value \\
\hline $\mathrm{A}_{11}$ & --- & --- & 1.1328 & 0.94188 \\
\hline $\mathrm{A}_{22}$ & 1.2134 & 0.96068 & --- & --- \\
\hline $\mathrm{A}_{33}$ & 0.4205 & 0.23067 & 0.6701 & 0.42470 \\
\hline $\mathrm{A}_{132}$ & 0.5187 & 0.21631 & --- & --- \\
\hline $\mathrm{A}_{131}$ & --- & --- & 0.2241 & 0.10165 \\
\hline $\mathrm{A}_{232}$ & 0.6298 & 0.24986 & --- & --- \\
\hline
\end{tabular}

Table 1 shows the comparison of the Hamaker constants and coefficients for the positive and negative sputum samples. $A_{11}$ is Hamaker constant for the uninfected sputum samples. $A_{22}$ is the Hamaker constant for the M-Tb, here represented by the infected macrophage. This is as a result of no known process of isolation of the $\mathrm{M}-\mathrm{Tb}$ at the moment. This is a very close approximation for the bacteria owing to the manner of the infection mechanism. The Hamaker constants $A_{33}$ for the sputum show greater values for the uninfected samples which regularly indicate a higher surface energy than the infected samples. The higher absolute values of $A_{132}$ and $A_{232}$ as against that of $A_{131}$, as well as the lower value of the absolute combined Hamaker coefficient $A_{131 a b s}$ for the uninfected samples is a clear 
Chukwuneke J. L., Achebe C. H., Ejiofor O. S. and Sinebe J. E.; Surface Energetics of Mycobacterium Tuberculosis-Macrophage Interactions. Journal of Biomedical Engineering and Medical Imaging, Volume 2, No 6, December (2015), pp 49-61

suggestion of the relevance of the concept of Hamaker coefficient in the M-Tb infection process. The surface energy $A_{131}$ of the macrophages is less than the surface energy $A_{232}$ of the bacteria (M-Tb).

Table 2: Values of the Hamaker Constants and Hamaker Coefficients for the Infected and Uninfected M-TB/HIV Sputum Samples

\begin{tabular}{|c|c|c|c|c|}
\hline $\begin{array}{c}\text { Variable } \\
\left(\times 10^{-21} \text { Joule }\right)\end{array}$ & \multicolumn{2}{|c|}{ Infected Sputum } & \multicolumn{2}{c|}{ Uninfected Sputum } \\
\hline & $\begin{array}{c}\text { Peak } \\
\text { Value }\end{array}$ & $\begin{array}{c}\text { Absolute } \\
\text { Value }\end{array}$ & Peak Value & Absolute Value \\
\hline$\tilde{A}_{11}$ & --- & --- & 1.1328 & 0.94188 \\
\hline$\widetilde{A}_{22}$ & 1.0267 & 0.97862 & --- & --- \\
\hline$\widetilde{A}_{33}$ & 0.5962 & 0.28812 & 0.6701 & 0.42470 \\
\hline$\tilde{A}_{132}$ & 0.4253 & 0.18825 & --- & --- \\
\hline$\tilde{A}_{131}$ & --- & --- & 0.2241 & 0.10165 \\
\hline$\tilde{A}_{232}$ & 0.5014 & 0.20474 & --- & --- \\
\hline
\end{tabular}

Comparing tables 1 and 2; $A_{33}$, which serves as the energy of sputum as an intervening medium, is seen in $\mathrm{M}-\mathrm{Tb}$ data to be reduced by infection from $0.4247 \times 10^{-21} \mathrm{~J}$ to $0.23067 \times 10^{-21} \mathrm{~J}$ by a factor of about $45.7 \%$ (see table 1). In M-Tb/HIV co-infection, the reduction is from $0.4247 \times 10^{-21} \mathrm{~J}$ to $0.28812 \times 10^{-21} \mathrm{~J}$, a factor of about $32.2 \%$ (see table 2). The reduction is lower in M-Tb/HIV co-infection probably because of the interaction between HIV and Tb. For the combined Hamaker coefficient, the value is $0.21631 \times 10^{-21} \mathrm{~J}$ for $\mathrm{M}-\mathrm{Tb}$ and $0.18825 \times 10^{-21} \mathrm{~J}$ for M-Tb/HIV. This result is as expected. HIV has the tendency to reduce the energy on the surface of a given material, in this case by about $13 \%$, conforming adverse effects observed in HIV patients with tuberculosis. Note that the values of $A_{132}$ are all positive showing that attraction exists between the macrophage and the M-Tb particles. The effect of the infection can only be abated if a drug, in the form of additive is added that can change the value of $A_{132}$ to negative under that condition, mutual repulsion will occur and it will be expected that, in principle, the Tb bacteria will not attack the macrophage.

\section{Conclusion}

This research predicts the interaction that occurs between M-Tb/HIV co-infections and macrophages. This prediction was based on the concept of van der waals attractive forces and absolute Hmaker coefficient whose positive values indicate attraction. The positive values of the absolute combined Hamaker coefficients $\mathrm{A}_{132}=0.21631 \times 10^{-21}$ Joule and $\tilde{\mathrm{A}}_{132}=0.18825 \times 10^{-21}$ Joule obtained for both the M-Tb and $\mathrm{M}-\mathrm{Tb} / \mathrm{HIV}$ positive samples respectively, are a confirmation that the sputum samples were actually infected. The absolute Hamaker coefficient $A_{131}=0.10165 \times 10^{-21}$ Joule gives the interaction energy among the macrophage cells in the sputum while $A_{232}$ is the interaction energy among the Tb particles in the sputum. $A_{232}$ for M-Tb/HIV co-infection is less than that for M-Tb alone. Reduction in energy in the presence of HIV confirms the adverse effect when Tb and HIV occur simultaneously in a patient. Reduction in energy leads to reduction in macrophage cells in M-Tb/HIV patient and hence greater prospect for death. This is so since a positive Hamaker value for any interacting system implies an attraction between the interacting bodies or particles.

This work concludes that there is a prospect of finding remedy for the M-Tb/HIV pandemic since the desired outcome is that the bacteria do not adhere to the macrophage to avoid penetration, in which case a condition for rendering combined Hamaker coefficient negative is required. Thus, a condition was 
sought for repulsion to occur and that condition will be based on the value of $A_{33}$ that would render the absolute combined Hamaker coefficient $A_{132 a b s}$ negative. To achieve the condition of $A_{33}$ above, possible additive(s) to the system (in form of drugs) to the sputum as intervening medium should be required. That, as expected, may be the much desired way out for drug resistant strains of the M-Tb bacteria.

\section{REFERENCES}

[1] Adeeb S., Gauhar R., Mazhar U., Waleed AK, Young SL., (2013). Challenges in the development of drugs for the treatment of tuberculosis. The Brazilian journal of infectious diseases, 17(1): $74-81$.

[2] Charles, Kittel, (1996): Introduction to Solid state Physics, $7^{\text {th }}$ Ed., John Willey and sons Inc. New York. 308.

[3] Chukwuneke J.L., Achebe C.H., Omenyi S.N. (2015). Mycobacterium Tuberculosis (M-TB)-Human Sputum Interaction Mechanisms Energetics. International Journal of Biological Engineering, 5(2): $23-30$.

[4] Corbett El, Marston B. et al., (2006). Tuberculosis in Sub-Sahara Africa: opportunities, challenges and changes in the era of antiretroviral treatment. Lancert, 367: 926-937.

[5] De Souza M. V. N., (2006). Recent patents on Anti-infective. Drug Discovery, 1: 33 - 34.

[6] Gonzalez-Juarrero M., Turner O. C., Turner J., Marietta P., Brooks J. V., Orme I. M., (2001). Temporal and Spatial arrangement of lymphocytes within lung granulomas induced by aerosol infection with mycobacterium tuberculosis. Infect. Immun. 69: 1722-1728.

[7] Hamaker, H.C., (1937). The London - Van der Waals attraction between spherical Particles. Physica, 4: 1058.

[8] Hough D. H and White L. R., (1987). Adv Colloid Interface. Science, 28: 35.

[9] Lifshitz E. M., (1961). The Theory of Molecular Attractive Forces between Solids. Advanced Physics. 10: $165-209$.

[10] Maartens G., Wilkinson R. J., (2007). Tuberculosis. Lancert, 370: 2030 - 2043.

[11] Nunes J. E. S., Ducati R. G., Breda A., Rosado L. A., De Souza B. M., Palma M. S., Santos D. S., Basso L. A., (2011). Molecular, kinetic Thermodynamic and Structural analysis of mycobacterium tuberculosis hisDencoded metal-dependent dimeric histidinol dehydrogenase (EC1.1.1.23). Archives Biochemistry and Biophysics, 512: $143-153$.

[12] Nunn P., Willianms B. et al., (2005). Tuberculosis control in the era of HIV. Nat. Rev. Immunology, 5(10): $819-826$.

[13] Robinson, T.S., (1952). Optical Constants by Reflection. Proceedings of the Physical Society London 65(11): B910

[14] Visser, J., (1981). Advances in Interface Science, Elsevier Scientific Publishing Company, Amsterdam, 15: 157-169. 
Chukwuneke J. L., Achebe C. H., Ejiofor O. S. and Sinebe J. E.; Surface Energetics of Mycobacterium Tuberculosis-Macrophage Interactions. Journal of Biomedical Engineering and Medical Imaging, Volume 2, No 6, December (2015), pp 49-61

[15] World Health Organization (WHO), (2005). Tuberculosis, WHO Information: Fact Sheets. http://www.who.int/mediacentre/factsheets/fs104/en/index. html, Retrieved 24-11-13.

[16] World Health Organization (WHO), (2012). Global tuberculosis report 2012. Geneva, Switzerland. http://www.who.int/tb/publications/global_report/, Retrieved 24-11-13.

[17] World Health Organization (WHO), (2009). Global tuberculosis Control: Epidemiology, strategy, financing, WHO report 2009, Geneva, Switzerland, WHO/HTM/TB/2009.411. 